\title{
Intracystic papillary carcinoma of the male breast: a case report
}

\author{
Haruhito Kinoshita ${ }^{1}$, Shinichiro Kashiwagi ${ }^{2^{*}}$ (D), Hitoshi Teraoka ${ }^{1}$, Takuya Mori ${ }^{1}$, Kenji Kuroda ${ }^{1,2}$, Mikio Nanbara $^{1}$, \\ Eiji Noda', Takaaki Chikugo ${ }^{3}$, Kosei Hirakawa ${ }^{2}$ and Masaichi Ohira ${ }^{2}$
}

\begin{abstract}
Background: Intracystic papillary carcinoma (IPC) is defined as cancer that develops from the wall of a cyst in the breast. As breast cancer in men accounts for only $1 \%$ of all breast cancers, male IPC is an extremely rare form of the disease. The present case report examines IPC in a man, along with an in-depth literature discussion.

Case presentation: A 64-year-old Japanese man noticed a mass in the right breast and sought medical attention. An elastic and soft neoplastic 3-cm lesion was palpated in the right papilla. As a 1-cm solid tumor with a gradual rise from the cyst wall was confirmed within the cyst, vacuum-assisted biopsy (VAB) was performed on that site. Pathological examination of the biopsy revealed heterotypic cells with an enlarged oval nucleus forming dense papillary structures mainly of vascular connective tissue component. Contrast-enhanced computed tomography (CT) confirmed thickening of the wall that protruded outside the cyst. The preoperative diagnosis was right breast cancer (male IPC) TisNOMO stage 0 luminal B-like. Total mastectomy and sentinel lymph node biopsy were performed. In the excised specimen, a 4.0-cm unilocular cyst was found, along with a 1-cm solid tumor with a gradual rise from the cyst wall. Pathological diagnosis of the resected specimen shared similar characteristics with the solid tumor in the cyst: notably, an oval nucleus with histologically clear nucleolus and fine granular chromatin, cylindrically shaped heterotypic cells, and the presence of basophilic cells in the papillary growth with a thin stem of fibrovasculature as the axis. Some invasion of tumor cells into the interstitium was confirmed. As such, the final diagnosis was right breast cancer (male IPC) T2NOMO stage IIA luminal B-like. The expression of hormone receptor (ER and PgR) was high, and endocrine therapy was initiated postoperatively (20 mg/day tamoxifen). At the present time (3 months postoperation), there has not been any evidence of metastasis.
\end{abstract}

Conclusions: We reported a rare case of an IPC in the male breast, along with a literature review.

Keywords: Intracystic papillary carcinoma, Breast cancer, Male, Ultrasonography, Vacuum-assisted biopsy

\section{Background}

Intracystic papillary carcinoma (IPC) was first reported by Brodie in 1846, and its incidence is approximately $0.26-2.0 \%$ of all breast cancers [1]. IPC is defined as cancer that develops from the wall of a cyst in the breast. As breast cancer in men accounts for only $1 \%$ of all breast cancers [2], male IPC is an extremely rare form of the disease [3-5]. The present case report examines IPC in a man, along with an in-depth literature discussion.

\footnotetext{
* Correspondence: spqv9ke9@view.ocn.ne.jp

2Department of Surgical Oncology, Osaka City University Graduate School of

Medicine, 1-4-3 Asahi-machi, Abeno-ku, Osaka 545-8585, Japan

Full list of author information is available at the end of the article
}

\section{Case presentation}

A 64-year-old Japanese man noticed a mass in the right breast and sought medical attention. The patient's medical records showed that he was currently undergoing treatment for alcoholic hepatitis. Physical findings confirmed gynecomastia in both breasts. An elastic and soft neoplastic 3-cm lesion was palpated in the right papilla (Fig. 1a). There were no skin lesions, and swollen lymph nodes were not palpated. Blood tests showed that tumor marker levels were normal (carcinoembryonic antigen [CEA] level of $1.6 \mathrm{ng} / \mathrm{ml}$ [normal, $<5.0 \mathrm{ng} / \mathrm{ml}$ ], cancer antigen $15-3$ [CA15-3] level of $7.5 \mathrm{IU} / \mathrm{ml}$ [normal, $<25.0 \mathrm{IU} / \mathrm{ml}$ ], and National Cancer Center-Stomach-439 (NCC-ST-439) level of $1.0 \mathrm{U} / \mathrm{ml}$ [normal, $<4.5 \mathrm{U} / \mathrm{ml}]$ ). On mammary ultrasonography, a cystic lesion of $3.9 \times 2.6 \mathrm{~cm}$ was confirmed 

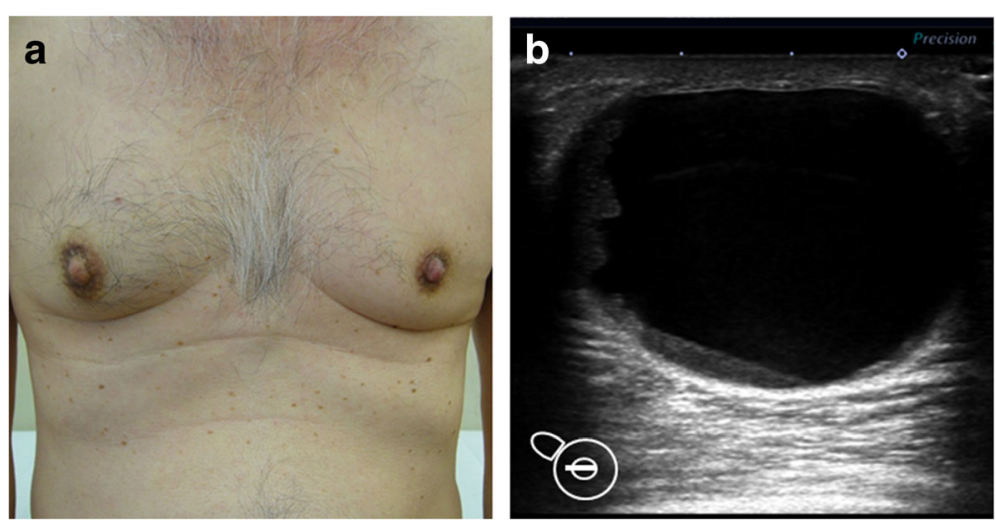

Fig. 1 Physical and ultrasonography findings: An elastic and soft neoplastic 3-cm lesion was palpated in the right papilla (a). On mammary ultrasonography, a cystic lesion of $3.9 \times 2.6 \mathrm{~cm}$ was confirmed immediately below the right papilla (mixed pattern). As a 1-cm solid tumor with a gradual rise from the cyst wall was confirmed within the cyst $(\mathbf{b})$

immediately below the right papilla (mixed pattern). As a 1 -cm solid tumor with a gradual rise from the cyst wall was confirmed within the cyst, vacuum-assisted biopsy (VAB) was performed on that site (Fig. 1b). Pathological examination of the biopsy revealed heterotypic cells with an enlarged oval nucleus forming dense papillary structures mainly of vascular connective tissue components (Fig. 2a, b). There was a nucleolus in the nucleus, and some mitotic images were noted. No invasion of tumor cells into the interstitium was noted. Upon immunohistochemical staining, estrogen receptor (ER) and progesterone receptor (PgR) were diffusely positive, human epidermal growth factor receptor 2 (HER2) was negative, and the Ki 67 index was $62.5 \%$. Contrast-enhanced computed tomography (CT) confirmed thickening of the wall that protruded outside the cyst (Fig. 3a, b). A systemic examination demonstrated that there were no metastases to the lungs, liver, or lymph nodes. Furthermore, there were no bone metastases as determined via bone scintigraphy. The preoperative diagnosis was right breast cancer (male IPC) TisN0M0 stage 0 luminal Blike. Total mastectomy and sentinel lymph node biopsy were performed. Intraoperative pathological diagnosis confirmed a lack of metastasis to the sentinel lymph node; hence, axillary lymph node dissection was omitted. In the excised specimen, a $4.0-\mathrm{cm}$ unilocular cyst was found, along with a $1-\mathrm{cm}$ solid tumor with a gradual rise from the cyst wall (Fig. 4a, b). Pathological diagnosis of the resected specimen shared similar characteristics with the solid tumor in the cyst: notably, an oval nucleus with histologically clear nucleolus and fine granular chromatin, cylindrically shaped heterotypic cells, and the presence of basophilic cells in the papillary growth with a thin stem of fibrovasculature as the axis. Some invasion of tumor cells into the interstitium was confirmed. In the papillary structure, p63-positive myoepithelial cells were absent and it was not biphasic (nuclear grade grade 2, nuclear atypia score 3, mitotic counts score 1) (surgical margin free) (Fig. 5a, b). ER and PgR staining were diffusely positive, HER2 staining
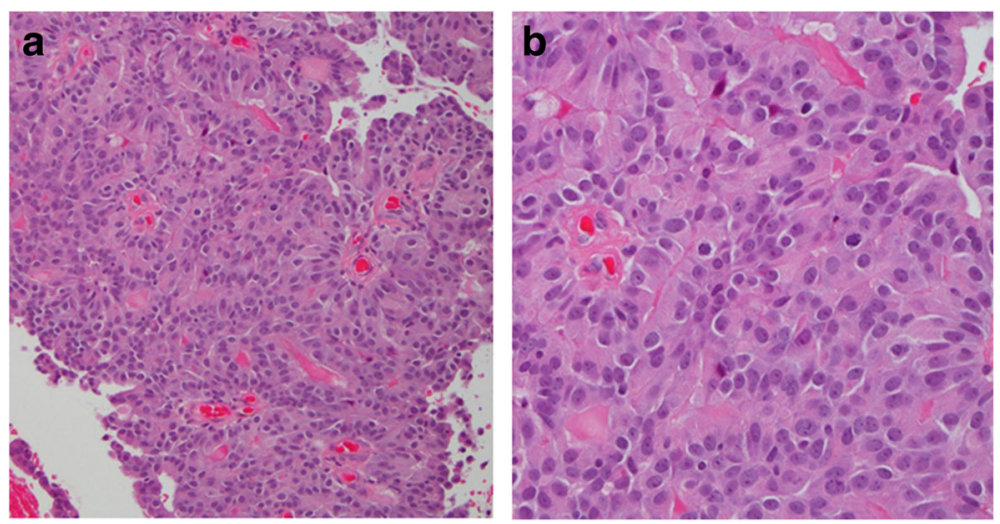

Fig. 2 Pathological findings of vacuum-assisted biopsy: Pathological examination of the biopsy revealed heterotypic cells with an enlarged oval nucleus forming dense papillary structures mainly of vascular connective tissue components $(\mathbf{a} \times 100)(\mathbf{b} \times 400)$ 


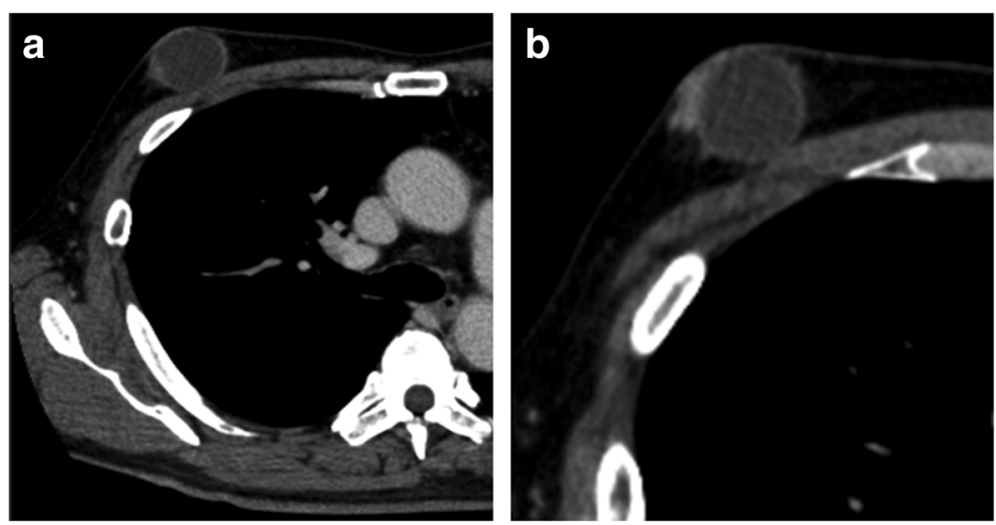

Fig. 3 CT image findings: Contrast-enhanced computed tomography confirmed thickening of the wall that protruded outside the cyst (a, b)

was negative, and the Ki 67 index was $6.6 \%$. In immunostaining, cytokeratin (CK) 20 was negative, and androgen receptor (AR) was positive. As such, the final diagnosis was right breast cancer (male IPC) T2N0M0 stage IIA luminal B-like. The expression of hormone receptor (ER and PgR) was high, and endocrine therapy was initiated postoperatively $(20 \mathrm{mg} /$ day tamoxifen $)$. At the present time (3 months postoperation), there has not been any evidence of metastasis.

\section{Discussion}

Breast cancers containing cysts can be divided into three types based on the tumor initiation site and pathological criteria: (1) necrosis at the center of noncystic breast cancers, leading to secondary cystoid degeneration (cystic breast cancer), (2) breast cancer and mammary cystic disease combined, where cancer has developed into mammary cysts and spread (combined breast cancer and cystic disease), and (3) breast cancer that has developed directly from the cystic wall; these tumors are considered IPC [6]. However, it is frequently difficult to make these distinctions, and since this particular case had a clear cystic structure and tumor was present within the cyst, it was formally diagnosed as IPC.

IPC is often discovered as an uneven mass, and without medical intervention, it tends to form relatively large tumor masses. When there is no invasion outside of the cyst wall, the malignancy is treated as Tis [7]. However, since there is a large amount of cystic components present in biopsy material, it is difficult to confirm invasion preoperatively [6]. Fineneedle aspiration cytology (FNAC) and core-needle biopsy (CNB) are usually performed, but the false negative results by cytology are relatively frequent [8]. In this case, we performed VAB to sample the rich biopsy material, but unfortunately could not confirm invasion preoperatively.

Since the prognosis of IPC after surgery is good and the incidence of local recurrence is low [9], if it is possible, partial mammary resection should be performed. However, as many cases present close to
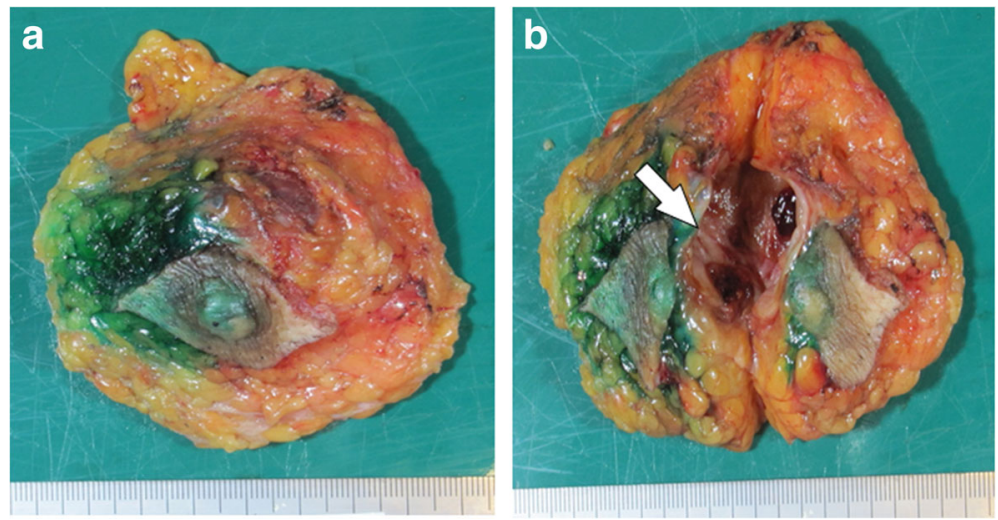

Fig. 4 Macroscopic diagnosis of the resected specimen: In the excised specimen, a 4.0-cm unilocular cyst was found, along with a 1-cm solid tumor with a gradual rise from the cyst wall (arrow) (a anterior image) (b split face of posterior image) 

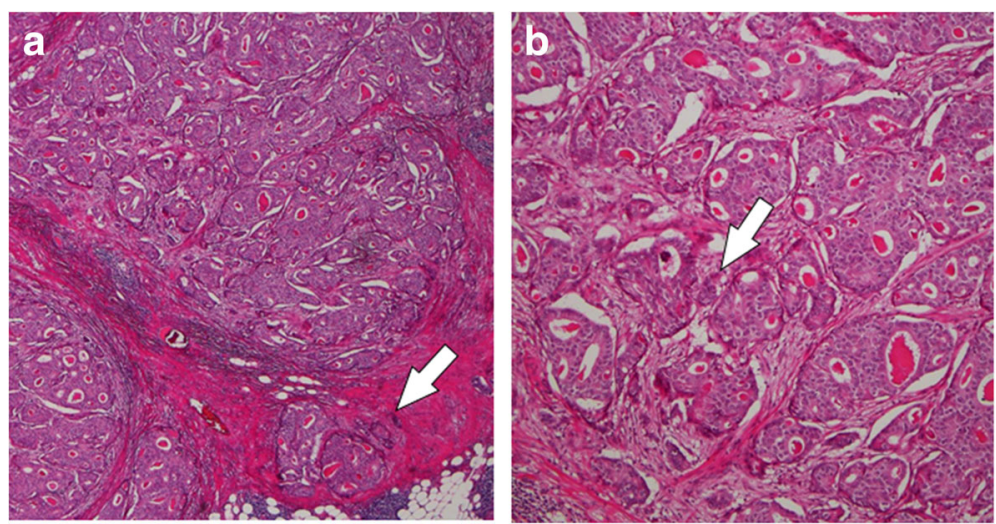

Fig. 5 Pathological diagnosis of the resected specimen: Pathological diagnosis of the resected specimen shared similar characteristics with the solid tumor in the cyst: notably, an oval nucleus with histologically clear nucleolus and fine granular chromatin, cylindrically shaped heterotypic cells, and the presence of basophilic cells in the papillary growth with a thin stem of fibrovasculature as the axis $(\mathbf{a} \times 100)(\mathbf{b} \times 400)$. Some invasion of tumor cells into the interstitium was confirmed (arrow)

the mammary glands [4], many physicians rely on total mastectomy, similar to that performed in this case $[4,10]$. With respect to the axillary operation, similar criteria to routine breast cancer surgery are used, and in this case, as there was no metastasis in the sentinel lymph node biopsy, axillary dissection was omitted.

Malignant progression in IPC is relatively low, with the majority of cases having a good prognosis, and there is no difference in prognosis between noninvasive and invasive types $[4,11]$. There is currently no standard of care considering postoperative treatment for IPC since there have not been many reported cases and prognosis following routine intervention based on tumor subtype is quite good. There are no clear guidelines on IPC management. Grabowski et al. confirmed that surgery is the mainstream of treatment [12]. The prognosis of IPC is excellent with low locoregional and distant recurrence rates, so mastectomy is usually not necessary, unless it is technically inevitable [12]. Moreover, there has been no clear indication for adjuvant endocrine therapy, even among the hormonereceptor-positive patients. If there is non-invasive ductal carcinoma outside the cyst wall or micro-invasive carcinoma in the interstitium, radiation therapy and pharmacotherapy are usually implemented, but these therapies for IPC have not been extensively evaluated [13]. However, similar to normal breast cancer, treatment for the intrinsic subtype should be performed, and as most male breast cancers are hormone receptor positive, tamoxifen is often administered [14, 15]. Also, postoperative adjuvant therapy for male breast cancer is thought that it should be done according to female breast cancer [16]. Indeed, the case presented here was hormone receptor positive, and tamoxifen was administered postoperatively [17-19].

\section{Conclusions}

In conclusion, we reported a rare case of an IPC in the male breast, along with a literature review.

\section{Abbreviations \\ AR: Androgen receptor; CA15-3: Cancer antigen 15-3; CEA: Carcinoembryonic antigen; CK: Cytokeratin; CNB: Core-needle biopsy; ER: Estrogen receptor; FNAC: Fine-needle aspiration cytology; HER2: Human epidermal growth factor receptor 2; IPC: Intracystic papillary carcinoma; NCC-ST-439: National Cancer Center-Stomach-439; PgR: Progesterone receptor; VAB: Vacuum- assisted biopsy}

\section{Acknowledgements}

We thank Yoshitaka Maekawa, Mako Kitano, Mayo Morikawa, Yoshimi Sugita, Akiko Sakamoto, and Hiroko Uono (Inspection Department, Baba Memorial Hospital) for helpful advice regarding ultrasound findings. This study was funded by grants from the Japan Society for the Promotion of Science (KAKENHI, Nos. 25461992, 26461957, and 17K10559) to Shinichiro Kashiwagi.

\section{Funding}

This study was supported in part by Grants-in Aid for Scientific Research (KAKENHI, Nos. 25461992, 26461957, and 17K10559) from the Ministry of Education, Science, Sports, Culture and Technology of Japan.

\section{Availability of data and materials}

The datasets supporting the conclusions of this article is included within the article.

\section{Authors' contributions}

All authors were involved in the preparation of this manuscript. HK collected the data, and wrote the manuscript. SK, HT, TM, KK, MN, and EN performed the operation and designed the study. TC perfomed pathological diagnosis. HK and SK summarized the data and revised the manuscript. KH and MO made substantial contribution to the study design, performed the operation, and revised the manuscript. All authors read and approved the final manuscript.

Ethics approval and consent to participate Not applicable.

\section{Consent for publication}

Written informed consent was obtained from the patient for publication of this case report and any accompanying images. A copy of the written consent is available for review by the Editor-in-Chief of this journal.

Competing interests

The authors declare that they have no competing interests. 


\section{Publisher's Note}

Springer Nature remains neutral with regard to jurisdictional claims in published maps and institutional affiliations.

\section{Author details}

'Department of Surgery, Baba Memorial Hospital, Higashi 4-244 Hamadera Funao-cho, Nishi-ku, Sakai, Osaka 592-8555, Japan. ${ }^{2}$ Department of Surgical Oncology, Osaka City University Graduate School of Medicine, 1-4-3

Asahi-machi, Abeno-ku, Osaka 545-8585, Japan. ${ }^{3}$ Department of Pathology, Kinki University Faculty of Medicine, Osaka-sayama, Osaka 589-8511, Japan.

\section{Received: 13 August 2017 Accepted: 12 January 2018}

\section{Published online: 23 January 2018}

\section{References}

1. Czernobilsky B. Intracystic carcinoma of the female breast. Surg Gynecol Obstet. 1967;124(1):93-8.

2. Anderson WF, Devesa SS. Breast carcinoma in men. Cancer. 2005;103(2): 432-3. author reply 433

3. Romics L Jr, O'Brien ME, Relihan N, O'Connell F, Redmond HP. Intracystic papillary carcinoma in a male as a rare presentation of breast cancer: a case report and literature review. J Med Case Rep. 2009;3:13.

4. Tochika N, Takano A, Yoshimoto T, Tanaka J, Sugimoto T, Kobayashi M, Matsuura K, Araki K, Ogawa Y, Moriki T. Intracystic carcinoma of the male breast: report of a case. Surg Today. 2001;31(9):806-9.

5. Muallaoglu S, Ozdemir E, Kutluay L. Intracystic papillary carcinoma of the breast in a male patient: a case report. Case Rep Med. 2012;2012:378157.

6. Collins LC, Carlo VP, Hwang H, Barry TS, Gown AM, Schnitt SJ. Intracystic papillary carcinomas of the breast: a reevaluation using a panel of myoepithelial cell markers. Am J Surg Pathol. 2006;30(8):1002-7.

7. Solorzano CC, Middleton LP, Hunt KK, Mirza N, Meric F, Kuerer HM, Ross MI, Ames FC, Feig BW, Pollock RE, et al. Treatment and outcome of patients with intracystic papillary carcinoma of the breast. Am J Surg. 2002;184(4):364-8.

8. Levine PH, Waisman J, Yang GC. Aspiration cytology of cystic carcinoma of the breast. Diagn Cytopathol. 2003;28(1):39-44.

9. Imoto $S$, Hasebe T. Intracystic papillary carcinoma of the breast in male: case report and review of the Japanese literature. Jpn J Clin Oncol. 1998;28(8):517-20.

10. Hu ZI, Liu C, Fisher PR, Cohen JA. Intracystic papillary carcinoma of the breast in a male patient. Rare Tumors. 2016:8(1):6050.

11. Carter D, Orr SL, Merino MJ. Intracystic papillary carcinoma of the breast. After mastectomy, radiotherapy or excisional biopsy alone. Cancer. 1983; 52(1):14-9.

12. Grabowski J, Salzstein SL, Sadler GR, Blair S. Intracystic papillary carcinoma: a review of 917 cases. Cancer. 2008;113(5):916-20.

13. Fayanju OM, Ritter J, Gillanders WE, Eberlein TJ, Dietz JR, Aft R, Margenthaler JA. Therapeutic management of intracystic papillary carcinoma of the breast: the roles of radiation and endocrine therapy. Am J Surg. 2007;194(4): 497-500.

14. Khan MH, Allerton R, Pettit L. Hormone therapy for breast cancer in men. Clin Breast Cancer. 2015;15(4):245-50.

15. Javidiparsijani S, Rosen LE, Gattuso P: Male breast carcinoma: a clinical and pathological review. Int J Surg Pathol. 2017;25(3):200-5.

16. Giordano $\mathrm{SH}$. A review of the diagnosis and management of male breast cancer. Oncologist. 2005;10(7):471-9.

17. Ribeiro G, Swindell R. Adjuvant tamoxifen for male breast cancer (MBC). Br J Cancer. 1992;65(2):252-4

18. Giordano SH, Perkins GH, Broglio K, Garcia SG, Middleton LP, Buzdar AU, Hortobagyi GN. Adjuvant systemic therapy for male breast carcinoma. Cancer. 2005;104(11):2359-64.

19. Fogh S, Hirsch AE, Langmead JP, Goldberg SI, Rosenberg CL, Taghian AG, Powell SN, Kachnic LA. Use of tamoxifen with postsurgical irradiation may improve survival in estrogen and progesterone receptor-positive male breast cancer. Clin Breast Cancer. 2011;11(1):39-45. 